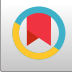

\title{
Prediction of the Course of Transient Tachypnea of the Newborn by Blood Laboratory Parameters at the Time of Admission
}

\author{
Sadrettin Ekmen (iD) ${ }^{1,}$ and Erkan Doğan ${ }^{2}$ \\ ${ }^{1}$ Neonatology Division, Department of Pediatrics, Faculty of Medicine, Karabuk University, Karabuk, Turkey \\ ${ }^{2}$ Department of Pediatrics, Faculty of Medicine, Karabuk University, Karabuk, Turkey \\ "Corresponding author: Fakulty of Medicine, Karabuk University, Karabuk, Turkey. Email: sadrettinekmen@hotmail.com \\ Received 2020 December 16; Revised 2021 March 08; Accepted 2021 April 10.
}

\begin{abstract}
Background: Transient tachypnea of the newborn (TTN) is a common but mild respiratory problem seen in late preterm and term newborns. However, it may sometimes cause severe morbidity.

Objectives: Therefore, biomarkers that can predict TTN severity may aid the clinician in determining the need for intensive care. Our aim was to identify whether blood gases and complete blood count parameters could be utilized to predict the severity of TTN.s Methods: We retrospectively examined the medical records of newborns ( $>37$ weeks gestation, > 2000 grams) diagnosed with TTN who were hospitalized in the Neonatal Intensive Care Unit (NICU) of Karabuk University Faculty of Medicine, Training and Research Hospital, Karabük/Turkey between June 2019 and June 2020. Patients were grouped according to the length of stay in the NICU. Group 1 included patients that did not require NICU monitoring after 48 hours, and group 2 included patients who continued to require NICU monitoring after 48 hours. Blood gases and complete blood count parameters were compared between the two groups.

Results: During the study period, 91 newborns were hospitalized in the NICU due to respiratory distress. Thirty-nine patients who did not meet the inclusion criteria were excluded. Gestational age and birth weight were significantly lower in group $2(\mathrm{P}<0.05)$. There were no significant differences between the groups in terms of leukocyte count, hemoglobin level, mean platelet volume (MPV), and platelet count. Partial carbon dioxide pressure $\left(\mathrm{PCO}_{2}\right)$ was significantly elevated, and base excess (BE) was significantly greater in group 2.

Conclusions: We determined a relationship between a prolonged stay in NICU and the levels of $\mathrm{PCO}_{2}$ and BE among patients hospitalized with a diagnosis of TTN. Therefore, $\mathrm{PCO}_{2}$ and $\mathrm{BE}$ values may be valuable to determine the prognosis of TTN in the early period. Despite being one of the most common causes of intensive care admission in the newborn population, there is limited data on the diagnosis, treatment, and prognosis of TTN; thus, we believe that our results will shed light on this problem.
\end{abstract}

Keywords: Blood Gas Analysis, Transient Tachypnea of the Newborn, Prognosis

\section{Background}

Transient tachypnea of the newborn (TTN) is a common respiratory problem after birth among late preterm and term newborns (1). It is seen with a frequency of approximately $3 \%-5 \%$ in infants (2). Although TTN usually regresses within 2 - 5 days, it may necessitate respiratory support via nasal continuous airway pressure (nCPAP) or mechanical ventilation (MV). In addition, it is also known as a cause of severe neonatal morbidities, such as pneumothorax and pulmonary hypertension (3). Preterm birth, low birth weight, macrosomia, cesarean delivery, perinatal asphyxia, male gender, maternal diabetes, and maternal asthma are risk factors associated with TTN development. Although the etiology of TTN is yet to be fully under- stood, it is thought to be associated with a delay in the resorption of fetal lung fluid. As such, delayed maturation of epithelial sodium channels, which are important for lymphatic resorption, has been suggested as an etiological factor (4). There is also some evidence indicating that immaturity and hypoxia may contribute to the pathophysiology in relation to their roles in the surfactant system (5-7). Markers that can predict the severity of TTN can assist clinicians in determining patients' requirements for advanced interventions or intensive care treatment.

\section{Objectives}

In this study, we aimed at determining whether blood gases and complete blood count (CBC) parameters could 
contribute to the prediction of TTN severity.

\section{Methods}

Our study was carried out by retrospectively examining the medical records of babies diagnosed with TTN who were monitored in the Neonatal Intensive Care Unit (NICU), between June 2019 and June 2020, in Karabuk University Faculty of Medicine, Training and Research Hospital, Karabük/Turkey. All newborns fulfilling the following criteria were included in the study: having a gestational age of 37 weeks, having a birth weight greater than $2000 \mathrm{~g}$, being diagnosed with TTN, and having blood gas and CBC results at the time of hospitalization.

The diagnosis of TTN was made according to the following clinical and laboratory criteria (8):

1) The onset of tachypnea (respiratory rate $>60$ beats/minute) within 6 hours after birth.

2) Tachypnea lasting for at least 12 hours.

3) Oxygen requirement more than $21 \%$.

4) Presence of at least one finding supporting TTN on chest X-ray (central vascular scars, signs of fluid, or hypermutation in interlobar fissures).

Patients with meconium aspiration syndrome, sepsis, chromosomal abnormalities, respiratory distress syndrome, pneumonia (detection of infiltration in chest radiography), congenital heart disease (detected by echocardiography), or stage 2/3 hypoxic-ischemic encephalopathy according to Sarnat and Sarnat (9), were excluded from the study.

As initial tests, $\mathrm{CBC}$, blood glucose, blood gas, and blood cultures were obtained from all hospitalized patients. Chest radiography was ordered at baseline and repeated when necessary. On the first postnatal day, 60 $\mathrm{mL} / \mathrm{kg}$ daily parenteral fluid was started.

Enteral feeding was minimally provided via orogastric catheter to babies whose respiratory rate fell below $60 / \mathrm{min}$ on the first day. While awaiting culture results, prophylactic antibiotic treatment was initiated according to the clinical sepsis protocol for patients with risk for sepsis.

Antibiotherapy was terminated as soon as possible (72 - 96 hours) in babies without growth in blood cultures whose acute phase reactants were within the reference range.

Oxygen saturation was continuously monitored by pulse oximetry to keep values between $90 \%$ and $98 \%$. Ventilation support was provided in the form of nasal continuous positive airway pressure (nCPAP) or nasal intermittent forced ventilation mode (NIMV) for patients with clinical grunting, retractions, and/or blood gas $\mathrm{pH}<7.25$ or partial carbon dioxide pressure $\left(\mathrm{PCO}_{2}\right)>60 \mathrm{mmHg}$. The files of patients diagnosed with TTN were evaluated in terms of demographic and clinical characteristics (gender, birth weight, gestational age, and delivery type). Patients' duration of stay in the NICU, laboratory results $\left(\mathrm{pH}, \mathrm{pCO}_{2}\right.$, base excess (BE), white blood cell count, platelet count, mean platelet volume (MPV), hemoglobin levels, and blood culture) and radiological examinations were also evaluated.

Blood samples were taken from the peripheral vein immediately after hospitalization and before any treatment was initiated. Blood samples for CBC were drawn into tubes containing ethylene diamine tetra-acetic acid (EDTA). CBC was analyzed using the CELL-DYN Ruby Hematology Analyzer (Abbott Diagnostics, Lake Forest, IL, USA). Blood gas was studied with the Siemens RAPID Point 500 Blood Gas Analyzer. CBC and blood gas were studied within 1 hour after birth.

The patients were grouped according to the length of stay in the NICU. Group 1 was defined as newborns in which NICU monitoring was not required after 48 hours, and group 2 included patients who continued to require NICU monitoring after 48 hours. The parameters analyzed in this study were compared between these two groups to determine whether they were associated with TTN severity.

Our study was approved by the Ethics Committee of Karabuk University Faculty of Medicine with the decision number of $2020 / 287$, dated $27 / 8 / 2020$. The study conformed to the Helsinki Declaration and good clinical practice guidelines.

\subsection{Ventilation Strategies}

Ventilator support with nCPAP or NIMV was applied with a newborn ventilator (SLE200, SLE Ltd, South Croydon, UK). For nCPAP, the positive end-expiratory pressure (PEEP) was set to $5 \mathrm{~cm} \mathrm{H}_{2} \mathrm{O}$. For NIMV, settings were as follows: $40 / \mathrm{min}$ rate, $20 \mathrm{~cm} \mathrm{H}_{2} \mathrm{O}$ peak inspiratory pressure (PIP), and $5 \mathrm{~cm} \mathrm{H}_{2} \mathrm{O}$ PEEP. The inspired oxygen fraction $\left(\mathrm{FiO}_{2}\right)$ was adjusted so that the oxygen saturation remained as $90 \%$ 98\% continuously. Treatment was continued with oxygen if saturation remained above $90 \%$ under the following conditions: when $\mathrm{FiO}_{2}$ need dropped below 0.3 (for nCPAP) or when $\mathrm{FiO}_{2}$ need dropped below 0.3 with $12 \mathrm{~cm} \mathrm{H}_{2} \mathrm{O}$ PIP (in NIMV). When the clinical signs of respiratory distress disappeared (grunting, retraction, tachypnea) and the oxygen saturation was $>90 \%$ without oxygen support, the TTN was considered to be improved.

\subsection{Statistical Methodology}

The SPSS software for Windows ${ }^{\circledR}$ version 26.0 (IBM Corporation, Armonk, New York, United States) was used for data analysis. The conformity of univariate data to 
normal distribution was evaluated by the KolmogorovSmirnov and Shapiro-Wilk tests, while variance homogeneity was evaluated with Levene's test. The receiver operating characteristics (ROC) analysis was utilized to determine whether the parameters that demonstrated a significant difference in univariate analyses (birth weight, gestational age, $\mathrm{PCO}_{2}$, and $\mathrm{BE}$ ) could be used to predict the severity of TTN. Cut-off points were identified with the Youden J index, and sensitivity/specificity values were calculated for each variable with regard to these thresholds. The odds ratio was used together with $95 \%$ confidence intervals to analyze the increase/decrease in likelihood. All analyses were performed at a 95\% confidence level, and a p-value of less than 0.05 was considered significant.

\section{Results}

During the study period, 91 newborns were hospitalized in the NICU due to respiratory distress. Thirty-nine patients who did not meet the inclusion criteria were excluded.

A final total of 52 newborns with TTN were divided into two groups according to their length of stay in the NICU. There were 23 patients in group 1 and 29 patients in group 2. Gender distribution and mode of delivery were similar between the groups. Gestational age was significantly lower in group $2(\mathrm{P}<0.05)$. Demographic and birth-related characteristics are shown in Table 1.

When the groups were compared with regard to laboratory findings, there was no significant difference between the two groups in terms of leukocyte count, hemoglobin level, MPV, and platelet count. PCO2 and BE were significantly higher in group 2 compared with group $1(\mathrm{P}<0.05)$ (Table 2).

Cut-off values for the detection of severe TTN (group 2) were identified for all parameters analyzed with ROC analysis. It was detected that birth weight had $96.6 \%$ sensitivity and $34.8 \%$ specificity with a cut-off value of 3490 grams. For gestational age, the 273-day threshold demonstrated $82.8 \%$ sensitivity and $47.8 \%$ specificity. For $\mathrm{PCO}_{2}$, the cut-off value was determined as 48.8 and had $87 \%$ sensitivity and $55.2 \%$ specificity. For BE, a -2.4 cut-off showed $86.2 \%$ sensitivity and $43.5 \%$ specificity (Table 3 ).

\section{Discussion}

In this study, we evaluated the possible relationships between TTN severity and parameters of CBC or blood gases on admission in newborns hospitalized for TTN.

There was a significant relationship between prolonged NICU need and increased $\mathrm{PaCO}_{2}$ and greater $\mathrm{BE}$ values in babies monitored with a diagnosis of TTN. Therefore, the determination of $\mathrm{PaCO}_{2}$ and BE levels in the early period may be useful in predicting the prognosis of TTN.

In addition, there was a positive correlation between the duration of intensive care support treatment and low birth weight and gestational week.

Although there have been numerous studies on markers related to the duration of tachypnea and its risk factors, to date, there is no consensus on markers for TTN. With regard to clinical factors, a previous study has shown that various antenatal risk factors, such as multiple pregnancies, placenta previa, preeclampsia, gestational diabetes, and premature rupture of the membranes were associated with tachypnea duration in TTN (10).

Other studies have shown that some biochemical markers, such as an atrial natriuretic peptide, B-type natriuretic peptide levels, and the lamellar body count in amniotic fluid can be used to estimate the severity of TTN (11-13). However, as mentioned before, there is no agreement on a clinical criterion or biochemical marker that indicates the severity of TTN. Such a biomarker can help physicians to predict the prognosis of the disease and facilitate early diagnosis, and appropriate use could lead to early intervention.

It is thought that asphyxia plays a role in the pathogenesis of TTN by causing pulmonary capillary leak syndrome -in relation to the fact that TTN is known to be a cause of hypoxia (14). The lower APGAR score in newborns with TTN supports the possible role of hypoxia in this condition (15); however, the APGAR score is affected by many other perinatal factors.

In two studies on TTN severity and hypoxia-related biomarkers, it has been shown that ischemia-related albumin, lactate, and lactate dehydrogenase levels are associated with the severity of $\operatorname{TTN}(16,17)$.

In another study, Oztekin et al. (18) reported that there was a positive correlation between a greater need for respiratory support in patients with TTN and the values of $\mathrm{pH}$, $\mathrm{PaO}_{2}$, and oxygen saturation, which are among the blood gas parameters measured in the first hour.

In our study, we observed that babies with TTN with elevated $\mathrm{PaCO}_{2}$ and greater $\mathrm{BE}$ were more likely to require prolonged NICU monitoring. Therefore, we think that these parameters can be used to predict the prognosis of TTN. We could not evaluate the relationship between $\mathrm{PaO}_{2}$ level and TTN severity because blood gas was taken from veins.

Kasap et al. (19) observed that tachypnea duration was negatively correlated with leukocyte count and hematocrit level. Ilhan and Bor (20) found that there was a positive relationship between the severity of TTN and low platelet count and platelet mass index. They attributed this to the possibility that low platelet count (or decreased function) would cause edema in the alveoli by increasing 


\begin{tabular}{|c|c|c|c|c|}
\hline & \multirow{2}{*}{ Overall $(\mathrm{N}=52)$} & \multicolumn{2}{|c|}{ Length of Stay in the NICU } & \multirow{2}{*}{ P-Value } \\
\hline & & $<48 \mathrm{~h}(\mathrm{~N}=23)$ & $\geq 48 \mathrm{~h}(\mathrm{~N}=29)$ & \\
\hline Gender & & & & $0,145 \mathrm{FE}$ \\
\hline Female & $11(21,2)$ & $7(30,4)$ & $4(13,8)$ & \\
\hline Male & $41(78,8)$ & $16(69,6)$ & $25(86,2)$ & \\
\hline Type of delivery & & & & $0,074 \mathrm{PE}$ \\
\hline CS & $40(76,9)$ & $15(65,2)$ & $25(86,2)$ & \\
\hline Spontaneous vaginal & $12(23,1)$ & $8(34,8)$ & $4(13,8)$ & \\
\hline Birth weight, $g$ & $3.015 \pm 493$ & $3.196 \pm 498$ & $2.872 \pm 448$ & $0,013 t$ \\
\hline Gestation, $d$ & $267(262,5-274,5)$ & $269(266-281)$ & $266(261-273)$ & $0,039 \mathrm{U}$ \\
\hline \multicolumn{5}{|c|}{$\begin{array}{l}\text { Abbreviations: FE, Fisher's exact test (exact); PE, Pearson chi-square test (exact); Q1, percentile 25\%; Q3, percentile 75\%; SD, standard deviation; } t \text {, independent sampl } \\
t \text {-test (Bootstrap); U, Mann Whitney U-test (Monte Carlo). } \\
{ }^{\mathrm{a} V a l u e s ~ a r e ~ e x p r e s s e d ~ a s ~ m e d i a n ~(Q 1-Q 3), ~ o r ~ m e a n ~} \pm \text { SD, or No. (\%). }\end{array}$} \\
\hline & \multirow{2}{*}{ Overall $(\mathrm{N}=52)$} & \multicolumn{2}{|c|}{ Length of Stay in the NICU } & \multirow{2}{*}{ P-Value } \\
\hline & & $<48 \mathrm{~h}(\mathrm{~N}=\mathbf{2 3})$ & $\geq 48 \mathrm{~h}(\mathrm{~N}=29)$ & \\
\hline PLT & $289,5(240 / 338,5)$ & $314(243 / 350)$ & $281(232 / 330)$ & $0,324 \mathrm{U}$ \\
\hline MPV & $9,55(9,1 / 10,1)$ & $9,3(8,9 / 10,1)$ & $9,7(9,1 / 10,1)$ & $0,706 \mathrm{U}$ \\
\hline $\mathrm{PaCO}_{2}$ & $47,88 \pm 12,38$ & $43,41 \pm 9,26$ & $51,42 \pm 13,50$ & $0,020 t$ \\
\hline $\mathrm{HCO}_{3}$ & $20,78 \pm 2,37$ & $21,35 \pm 2,80$ & $20,32 \pm 1,89$ & $0,153 t$ \\
\hline BE & $-4,12 \pm 3,06$ & $-3,12 \pm 3,52$ & $-4,91 \pm 2,42$ & $0,048 t$ \\
\hline $\mathbf{P h}$ & $7,30 \pm 0,09$ & $7,32 \pm 0,10$ & $7,29 \pm 0,09$ & $0,259 t$ \\
\hline HG & $18,02 \pm 2,24$ & $18,07 \pm 2,24$ & $17,97 \pm 2,27$ & $0,879 t$ \\
\hline WBC & $17,30 \pm 5,55$ & $16,32 \pm 3,31$ & $18,07 \pm 6,79$ & $0,223 t$ \\
\hline
\end{tabular}

Abbreviations: FE, Fisher's exact test (exact); PE, Pearson chi-square test (exact); SD, standard deviation; $t$, independent samples $t$-test (Bootstrap); U, Mann Whitney U-test (Monte Carlo).

${ }^{\mathrm{a}}$ Values are expressed as mean $\pm \mathrm{SD}$ or No. (\%).

membrane permeability (20). Contrary to these studies, we could not find a relationship between the severity of TTN and leukocyte count, hemoglobin level, platelet count, and mean platelet volume.

Although many clinical and laboratory studies have been conducted to predict the severity of TTN, there is no universally accepted biomarker yet. We think that a scoring system that can predict the prognosis of TTN can be developed by combining clinical and laboratory risk factors with larger prospective controlled studies. Previous studies have developed and reported some scoring systems that could be used in newborns and children with respiratory distress (21). Clues associated with disease prognosis have always been useful as supportive data points for physicians and also when informing patients and/or their relatives of the condition (22).

Early tachypnea and blood gas disorders in the early pe- riod are signs that the baby is going through mild hypoxia, which may adversely affect lung dynamics.

Considering that respiratory distress is the most common admission diagnosis among patients admitted to NICU, it is evident that TTN ranks among the most frequent causes necessitating NICU monitoring. Currently, there are no clear guidelines on clarification of the diagnosis, treatment, or prognosis of TTN. Therefore, we believe that our study will shed light on this problem.

The limitations of our study include the low number of cases due to drawing data from one center, the retrospective-observational design, and the lack of $\mathrm{PaO}_{2}$ assessments (because blood gas analysis was performed in venous samples). 


\begin{tabular}{|c|c|c|c|c|c|}
\hline \multirow{2}{*}{ Cut-off } & \multicolumn{2}{|c|}{ Duration in Intensive Care } & \multirow{2}{*}{ Odds Ratio (\%95 CI) } & \multirow{2}{*}{$\operatorname{AUC}(\mathrm{SE})$} & \multirow{2}{*}{ P-Value } \\
\hline & $<48 \mathrm{~h}$ & $\geq 48 \mathrm{~h}$ & & & \\
\hline Birth weight & & & & & 0,019 \\
\hline$<3490$ & $8(34,8) \mathrm{SP}$ & $1(3,4)$ & $14,9(1,7-130,9)$ OR & $0,677(0,075)$ & \\
\hline$\geq 3490$ & $15(65,2)$ & $28(96,6) \mathrm{SS}$ & & & \\
\hline Gestational day at birth & & & & & 0,034 \\
\hline$<273$ & $11(47,8) \mathrm{SP}$ & $5(17,2)$ & $4,4(1,2-15,6)$ OR & $0,667(0,078)$ & \\
\hline$\geq 273$ & $12(52,2)$ & $24(82,8) \mathrm{SS}$ & & & \\
\hline $\mathrm{PaCO}_{2}$ & & & & & 0,011 \\
\hline$\leq 48,8$ & $20(87,0) \mathrm{SP}$ & $13(44,8)$ & $8,2(2-33,8)$ OR & $0,692(0,075)$ & \\
\hline$>48,8$ & $3(13,0)$ & $16(55,2) S S$ & & & \\
\hline $\mathbf{B E}$ & & & & & 0,082 \\
\hline$<-2,4$ & $10(43,5) \mathrm{SP}$ & $4(13,8)$ & $4,8(1,3-18,3) \mathrm{OR}$ & $0,637(0,084)$ & \\
\hline$\geq-2,4$ & $15(56,5)$ & $25(86,2) \mathrm{SS}$ & & & \\
\hline
\end{tabular}

Abbreviations: AUC, area under the ROC curve; CI, confidence interval; SE, standard error; SS, sensitivity; SP, specificity.

${ }^{\mathrm{a}}$ Values are expressed as mean $\pm \mathrm{SD}$ or No. (\%).

${ }^{\mathrm{b}}$ Roc Curve analysis (Youden J index, Honley and McNell).

\section{Footnotes}

Authors' Contribution: Sadrettin Ekmen did conceptualization, formal analysis; investigation; methodology; project administration; software; supervision; visualization; writing-original draft; writing-review, and editing. Erkan Doğan did data curation; validation; software; writing- review, and editing.

Conflict of Interests: The authors declare no conflict of interest.

Ethical Approval: Our study was approved by the Ethics Committee of Karabuk University Faculty of Medicine with the decision number of 2020/287, dated 27/8/2020. The study conformed to the Helsinki Declaration and good clinical practice guidelines.

Funding/Support: This research did not receive any specific grant from funding agencies in the public, commercial, or not-for-profit sectors.

\section{References}

1. Alhassen Z, Vali P, Guglani L, Lakshminrusimha S, Ryan RM. Recent advances in pathophysiology and management of transient tachypnea of newborn. J Perinatol. 2020:1-11.

2. Hansen AK, Wisborg K, Uldbjerg N, Henriksen TB. Risk of respiratory morbidity in term infants delivered by elective caesarean section: cohort study. BMJ. 2008;336(7635):85-7. doi: 10.1136/bmj.39405.539282.BE. [PubMed: 18077440]. [PubMed Central: PMC2190264].

3. Yurdakok M, Ozek E. Transient tachypnea of the newborn: the treatment strategies. Curr Pharm Des. 2012;18(21):3046-9. doi: 10.2174/1381612811209023046. [PubMed: 22564298].
4. Helve O, Pitkanen O, Janer C, Andersson S. Pulmonary fluid balance in the human newborn infant. Neonatology. 2009;95(4):347-52. doi: 10.1159/000209300. [PubMed: 19494556].

5. Aslan E, Tutdibi E, Martens S, Han Y, Monz D, Gortner L. Transient tachypnea of the newborn (TTN): a role for polymorphisms in the beta-adrenergic receptor (ADRB) encoding genes? Acta Paediatr. 2008;97(10):1346-50. doi: 10.1111/j.1651-2227.2008.00888.x. [PubMed: 18540901].

6. Yurdakok M. Transient tachypnea of the newborn: what is new? J Matern Fetal Neonatal Med. 2010;23 Suppl 3:24-6. doi: 10.3109/14767058.2010.507971. [PubMed: 20807157].

7. Fiori HH, Henn R, Baldisserotto M, Bica IG, Fiori RM. Evaluation of surfactant function at birth determined by the stable microbubble test in term and near term infants with respiratory distress. Eur J Pediatr. 2004;163(8):443-8. doi: 10.1007/s00431-004-1473-2. [PubMed: 15185148].

8. Rawlings JS, Smith FR. Transient tachypnea of the newborn. An analysis of neonatal and obstetric risk factors. Am J Dis Child. 1984;138(9):869-71. doi: 10.1001/archpedi.1984.02140470067022. [PubMed: 6540983].

9. Sarnat HB, Sarnat MS. Neonatal encephalopathy following fetal distress. A clinical and electroencephalographic study. Arch Neurol.1976;33(10):696-705. doi: 10.1001/archneur.1976.00500100030012. [PubMed: 987769].

10. Tarcan A, Anuk D, Cindık N, Gürakan B. Risk factors for prolongation of disease in transient tachypnea of the newborn. Türkiye Klinikleri J Pediatrics. 2004;13:224-6.

11. Onal EE, Dilmen U, Adam B, Turkyilmaz C, Uysal FK, Oguz D. Serum atrial natriuretic peptide levels in infants with transient tachypnea of the newborn. J Matern Fetal Neonatal Med. 2005;17(2):145-9. doi: 10.1080/14767050400028550. [PubMed: 16076624].

12. Tsuda H, Takahashi Y, Iwagaki S, Uchida Y, Kawabata I, Hayakawa M, et al. Amniotic lamellar body counts can predict the occurrence of respiratory distress syndrome as well as transient tachypnea of the newborn (TTN). J Perinat Med. 2011;39(3):245-50. doi: 10.1515/jpm.2011.006. [PubMed: 21314236]. 
13. Aydemir O, Aydemir C, Sarikabadayi YU, Altug N, Erdeve O, Uras N, et al. The role of plasma N-terminal pro-B-type natriuretic peptide in predicting the severity of transient tachypnea of the newborn. Early Hum Dev. 2012;88(5):315-9. doi:10.1016/j.earlhumdev.2011.08.026. [PubMed: 21955499].

14. Perez Molina JJ, Romero DM, Ramirez Valdivia JM, Corona MQ. [Transient tachypnea of the newborn, obstetric and neonatal risk factors]. Ginecol Obstet Mex. 2006;74(2):95-103. Spanish. [PubMed:16637378].

15. Takaya A, Igarashi M, Nakajima M, Miyake H, Shima Y, Suzuki S. Risk factors for transient tachypnea of the newborn in infants delivered vaginally at 37 weeks or later. J Nippon Med Sch. 2008;75(5):269-73. doi: 10.1272/jnms.75.269. [PubMed: 19023165].

16. Oztekin O, Kalay S, Tayman C, Namuslu M, Celik HT. Levels of ischemiamodified albumin in transient tachypnea of the newborn. Am JPerinatol. 2015;30(2):193-8. doi: 10.1055/s-0034-1381319. [PubMed: 24915561].

17. Ozkiraz S, Gokmen Z, Boke SB, Kilicdag H, Ozel D, Sert A. Lactate and lactate dehydrogenase in predicting the severity of transient tachypnea of the newborn. J Matern Fetal Neonatal Med. 2013;26(12):1245-8. doi: 10.3109/14767058.2013.776532. [PubMed: 23414515].
18. Oztekin O, Kalay S, Tezel G, AkÇakuş M, Oygür N. Can we predict the duration of respiratory support in transient tachypnea of the newborn? Turk J Med Sci. 2012;42(Sup. 2):1494-8.

19. Kasap B, Duman N, Ozer E, Tatli M, Kumral A, Ozkan H. Transient tachypnea of the newborn: predictive factor for prolonged tachyp nea. Pediatr Int. 2008;50(1):81-4. doi: 10.1111/j.1442-200X.2007.02535.x. [PubMed: 18279211].

20. Ilhan O, Bor M. Platelet mass index and prediction of severity of transient tachypnea of the newborn. Pediatr Int. 2019;61(7):697-705. doi: 10.1111/ped.13899. [PubMed: 31140210].

21. Armangil D, Yurdakok M, Korkmaz A, Yigit S, Tekinalp G. Inhaled beta-2 agonist salbutamol for the treatment of transient tachypnea of the newborn. J Pediatr. 2011;159(3):398-403 e1. doi: 10.1016/j.jpeds.2011.02.028. [PubMed: 21481414].

22. Kahvecioglu D, Cakir U, Yildiz D, Alan S, Erdeve O, Atasay B, et al. Transient tachypnea of the newborn: are there bedside clues for predicting the need of ventilation support? Turk J Pediatr. 2016;58(4):400-5. doi: 10.24953/turkjped.2016.04.009. [PubMed: 28276213]. 